http://jmscr.igmpublication.org/home/ ISSN (e)-2347-176x ISSN (p) 2455-0450 crossref DOI: https://dx.doi.org/10.18535/jmscr/v10i1.10

\author{
Journal Of Medical Science And Clinical Research \\ IGM Publication \\ An Official Publication of IGM Publication
}

\title{
A Case Report of Non Food Grade Ethyl Alcohol Toxicity
}

\author{
Authors \\ Dr Snigdha Gannamraju", Dr K. Sai Abhinandan², Dr A. Rama Ratnam³, \\ Dr Prachee Nagrale ${ }^{4}$ \\ ${ }^{1}$ Senior Resident, Department of Ophthalmology, Mamata Medical College, Khammam \\ ${ }^{2}$ Post Graduate, Department of Ophthalmology, Mamata Medical College, Khammam \\ ${ }^{4}$ Professor, Mamata Medical College, Khammam
}

\section{Introduction}

Ethanol is a highly water soluble and low molecular weight compound that rapidly crosses cell membrane.

Absorption- occurs mainly in proximal intestinal tract i.e stomach $70 \%$, duodenum $25 \%$, small percentage in remaining intestinal tract. ${ }^{(1)}$

Metabolism- gastric alcohol dehydrogenase $(\mathrm{ADH})$ is responsible for $10 \%$ of alcohol metabolism i.e first pass metabolism. Remaining 90\% of ingested alcohol is metabolised to acetaldehyde along 3 liver enzymatic pathways i.e liver $\mathrm{ADH}$, microsomal ethanol oxidising system (MEOS) and catalase. ${ }^{(2)}$

Food or fluid in the gastric lumen dilutes ethanol concentration and reduces the efficiency of absorption. Caloric density and composition of a meal influence rate of gastric emptying. ${ }^{(3)}$

Distribution- completely miscible in water, insoluble in fat. Volume of distribution is proportional to lean body mass because adipose tissue contains little water. Therefore females have smaller volume of distribution. ${ }^{(4)}$

Elimination- $95-98 \%$ of ingested ethanol is metabolised in the liver first by enzyme alcohol dehydrogenase to acetaldehyde and then by the enzyme aldehyde dehydrogenase to acetic acid which dissociates to carbondioxide and water. ${ }^{(5)}$

\section{Case Report}

A 45 year old male patient presented to ophthalmology department with history of sudden painless diminution of vision both eyes since 4 days. No history of trauma and ocular surgery. No other ocular complaints. No known systemic illness.

On ocular examination visual acuity PL (+) PR inaccurate in both eyes with mid dilated nonreactive pupils in both eyes. Anterior segment findings were unremarkable and IOP recorded as $13 \mathrm{~mm}$ hg in RE and $16 \mathrm{~mm}$ hg in LE with AT. Extra ocular movements free and full range in all directions.

Dilated ophthalmoscopic examination revealed cup disc ratio within normal limits, optic disc oedema, and disc hyperaemia with tortuous and dilated vessels in both eyes suggestive of optic neuritis. Normal foveal reflex maintained.

Detailed history revealed consumption of sanitiser as an addiction on daily basis consuming $30 \mathrm{ml}$ twice a day over a period of 45 days.

CT brain plain study was suggestive of normal extraocular muscles, optic nerve normal 
bilaterally, no evidence of space occupying lesions, orbits normal in structure and configuration.

MRI brain T2 weighted image showed high signal abnormalities in bilateral basal ganglia, frontal, occipital sub cortical white matter consistent with oedematous and demyelination changes.

\section{Result}

Basing on history and ocular findings diagnosis of non food grade ethyl alcohol toxicity along with unknown flavouring agents was made.
Patient was administered intramuscular injections of 500mcg vitamin B12 alternate day for first one week followed by weekly single dose for next 11 weeks. Oral steroids in dosage of 40mg was initiated and gradually tapered over a period of 5 weeks along with strict prohibition of intake of ethylalcohol.

After 3 months visual acuity improved to counting fingers 4 metres in $\mathrm{RE}$ and counting fingers 3 metres in LE.

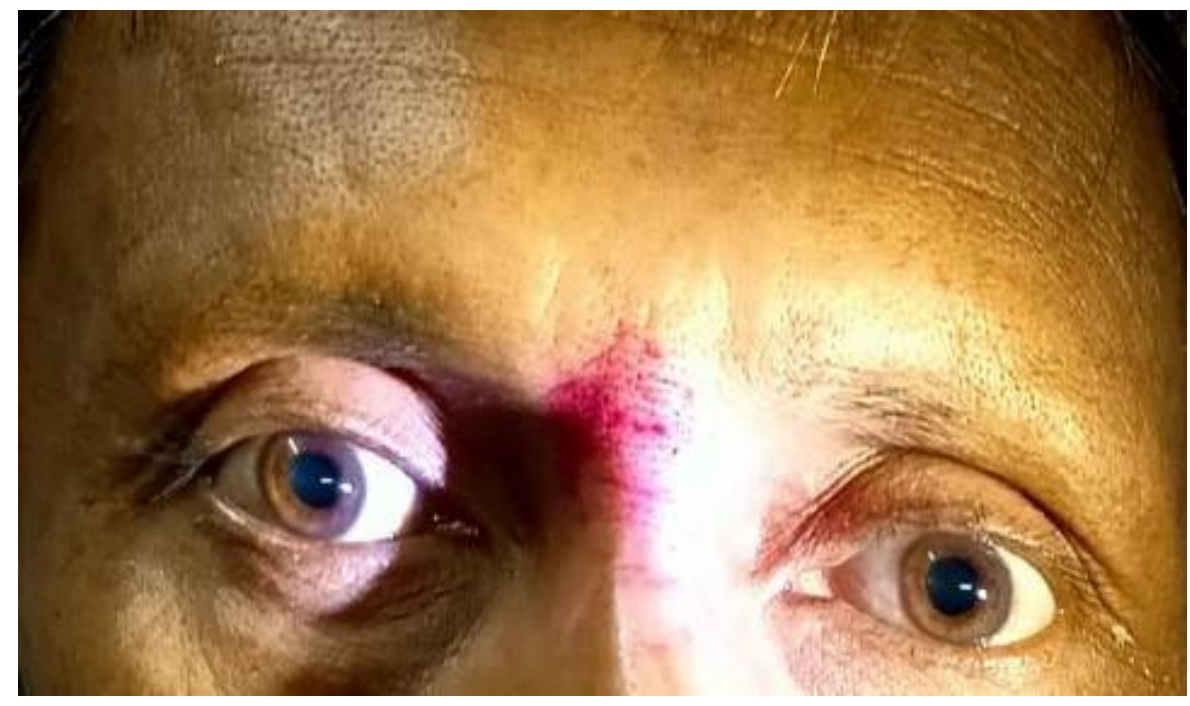

Mid Dilated Non Reactive Pupils in Right Eye and Left Eye
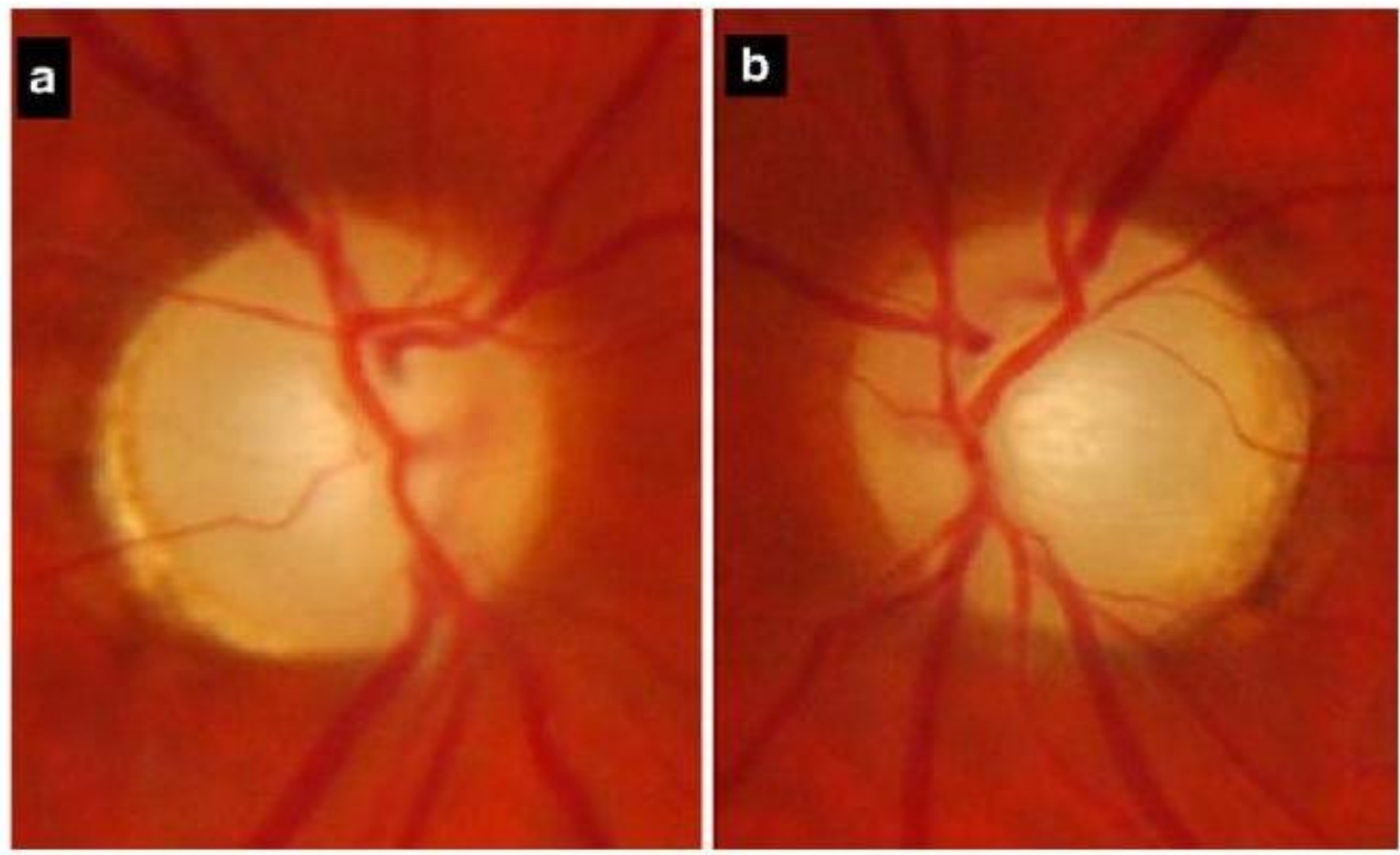

Dilated Ophthalmoscopic Picture of Right Eye and Left Eye Demonstrating Optic Disc Oedema and Dilated Tortuous Vessels in Both Eyes 


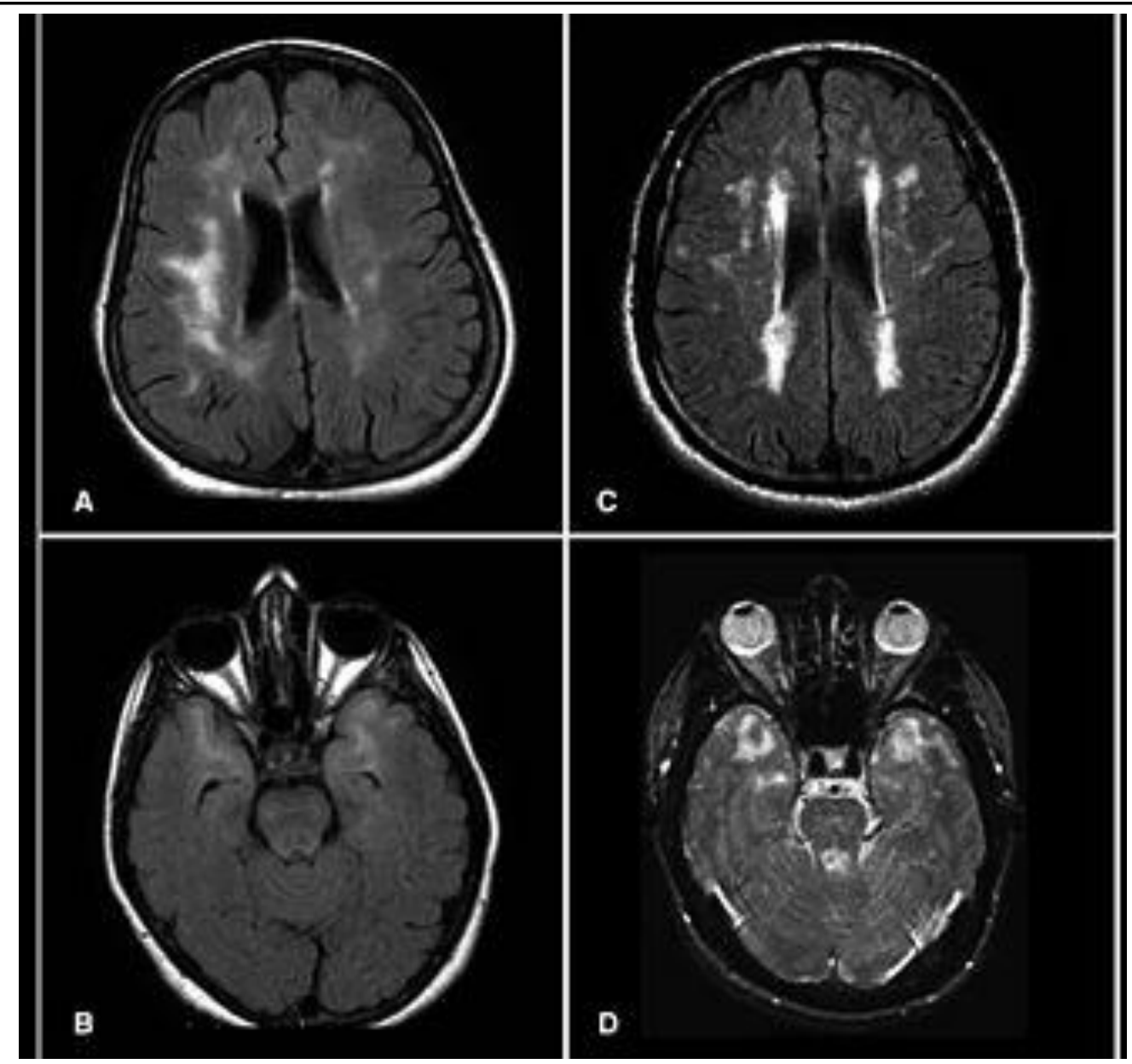

MRI Brain Plain Revealed Multiple Areas of Hyper intensities

\section{Discussion}

Alcohol Based Hand Sanitizer Consumption as an Emerging Public Health Concern

Alcohol based hand sanitizer is a liquid, gel or foam that contains ethanol or isopropanol used to disinfect hands. Hand hygiene is an important component of response to the emergence of SARS COV-2, the virus that causes corona virus disease COVID 19.

CDC recommends the use of alcohol based sanitizers that contain atleast $60 \%$ ethyl alcohol. ${ }^{(6)}$ According to FDA which regulates alcohol based hand sanitizers as an over the counter drug, methanol is not an acceptable ingredient.

Young children might unintentionally swallow these products whereas adolescents and adults with history of alcohol use disorder might intentionally swallow these products as an alcohol (ethanol) substitute. ${ }^{(7)}$

Hand sanitizer formulations vary by manufacturers and contain different concentrations of ethanol or isopropanol as well as additional potential ingredients such as acetone, 1propanol, 2- propanol, benzyl alcohol, hydrogen peroxide, glycerin, water and different perfumes. $(8,9)$

Clinical features include gastrointestinal manifestations as nausea, vomiting, abdominal pain, peptic ulcer, pancreatitis. Dysfunction of oesophageal, gastric, duodenal motility can lead to diarrhoea, hyponatremia.

Several metabolic alterations include hypoglycemia, lactic acidosis, hypokalemia, hypomagnesemia, hypocalcemia, hypoalbuminemia, hypophosphatemia. ${ }^{(1)}$

Cardiovascular manifestations include tachycardia, peripheral vasodilatation, volume depletion, hypotension.

Vision loss can range from mild to severe threatening. Others include centrocaecal scotoma, temporal pallor of the disc. The by products of alcohol metabolism along with the nutritional 
deficiencies blocks the electron transport within the mitochondria thereby decreasing production of ATP. Neurons with very low, very thin axons or unmyelinated nerve fibres are more prone to damage.

Severe life threatening consequence is the respiratory depression.

\section{Conclusion}

In addition to social distancing and consistent use of face masks, hand hygiene is important component of response to the emergence of SARS COV-2.

Safety messaging to avoid ingestion of any alcohol based hand sanitizer products should continue. They should only be used to disinfect hands and never be swallowed.

Here, the patient didn't require any intubation or haemodynamic support during his visit to the hospital.

Basing on history, alcohol dependence was noted and the psychiatrist recommended transfer to psychiatry faculty after clearance from medical and ophthalmic personnel for treatment of his psychiatry illness and alcohol dependence.

Consumers who have been exposed to alcohol based hand sanitizers should stop using them immediately and seek medical attention if they experience concerning symptoms.

Health department in all the states should coordinate with poison centres to identify cases of alcohol based hand sanitizer poisoning.

\section{Declaration of Patient Consent}

The authors certify that they have obtained all appropriate patient consent forms. The patient has given their consent for their images and other clinical information to be reported in the journal. However their name and initial will not be published.

Financial Support and Sponsorship- Nil

Conflicts of Interest- There are no Conflicts of Interest.

\section{References}

1. Marco CA, Kelen GD. Acute intoxication. Emerg Clin North Am 1990;8:731-48

2. Lieber CS. Hepatic metabolic and toxic effects of ethanol: 1991 uptake. Alcohol Clin Exp Res 1991;15:573-92.

3. Calbet JA, MacLean DA: Role of caloric content on gastric emptying in humans. J Physiol 498:553-9,1997.

4. Cowan JM, Weathermon A, McCutcheon JR, et al: Determination of volume of distribution for ethanol in male and female subjects. J Anal Toxicol 20:287-90,1996.

5. Holford NH: Clinical pharmacokinetics of ethanol .Clin Pharmacokinet 13:273-92, 1987.

6. CDC. Hand hygiene recommendations. Guidance for healthcare providers about hand hygiene and COVID-19. Atlanta, GA:US Department of Health and Human Services, CDC;2020. https://www.cdc.gov/coronavirus/2019ncov/hcp/hand-hygiene.html.

7. Schneir AB, Clark RF. Death caused by ingestion of an ethanol based hand sanitizer. J Emerg Med 2013; 45:358-60. https://doi.org/10.1016/j.jemermed.2013.0 3.018 .

8. Gormley NJ, Bronstein AC, Rasimas JJ, et al. The rising incidence of intentional ingestion of ethanol containing hand sanitizers. Crit Care Med. 2012:40(1):290$294 . \quad$ doi $10.1097 / \mathrm{CCM}$. Obo13e31822f09c0.

9. Archer JR,Wood DM, Tizzard Z, Jones AL, Dargan PI. Alcohol hand rubs: hygiene and hazards. BMJ. 2007;335 (7630):1154-1155.

10. Burbige EJ, Lewis Jr DR, Halsted CH. Alcohol and the gastrointestinal tract. Med Clin North Am 1984;68:77-89. 